PROCEEDINGS OF THE

AMERICAN MATHEMATICAL SOCIETY

Volume 132, Number 8, Pages 2377-2387

S 0002-9939(04)07410-6

Article electronically published on February 26, 2004

\title{
DENSITY OF IRREGULAR WAVELET FRAMES
}

\author{
WENCHANG SUN AND XINGWEI ZHOU
}

(Communicated by David R. Larson)

\begin{abstract}
We show that if an irregular multi-generated wavelet system forms a frame, then both the time parameters and the logarithms of scale parameters have finite upper Beurling densities, or equivalently, both are relatively uniformly discrete. Moreover, if generating functions are admissible, then the logarithms of scale parameters possess a positive lower Beurling density. However, the lower Beurling density of the time parameters may be zero. Additionally, we prove that there are no frames generated by dilations of a finite number of admissible functions.
\end{abstract}

\section{INTRODUCTION}

Wavelet systems that form frames for $L^{2}(\mathbb{R})$ have a wide variety of applications. An important problem in practice is therefore to determine conditions for wavelet systems to be frames. Many results, including necessary conditions and sufficient conditions, have been established during the past ten years. For example, see 3 [10], 12]-13], 15], 17]-19], and [21. In [4, Christensen, Deng and Heil studied the density of Gabor frames and proved that for a Gabor system $\left\{e^{i b_{n} x} g\left(x-a_{n}\right): n \in\right.$ $\mathbb{Z}\}$ to be a frame for $L^{2}(\mathbb{R})$, the time-frequency parameters $\left(a_{n}, b_{n}\right)$ must possess a lower Beurling density no less than $\frac{1}{2 \pi}$. For the case of wavelet systems, however, no similar result has been found.

In this paper, we study density conditions for irregular multi-generated wavelet systems of the form $\left\{s_{\ell, j}^{1 / 2} \psi_{\ell}\left(s_{\ell, j} \cdot-t_{\ell, k}\right): j \in \mathbb{J}_{\ell}, k \in \mathbb{K}_{\ell}, 1 \leq \ell \leq r\right\}$ to be frames, where $r$ is a fixed positive integer, $\psi_{\ell} \in L^{2}(\mathbb{R}), s_{\ell, j}>0, t_{\ell, k} \in \mathbb{R}$ and $\mathbb{J}_{\ell}, \mathbb{K}_{\ell} \subset \mathbb{Z}$. We call $s_{\ell, j}$ scale parameters and $t_{\ell, k}$ time parameters for a wavelet system. For any $1 \leq \ell \leq r$, let $S_{\ell}=\left\{s_{\ell, j}: j \in \mathbb{J}_{\ell}\right\}$ and $T_{\ell}=\left\{t_{\ell, k}: k \in \mathbb{K}_{\ell}\right\}$. Since $S_{\ell}$ and $T_{\ell}$ are sequences, repetitions of points are allowed. Let $S=\left\{s_{\ell, j}: 1 \leq \ell \leq r, j \in \mathbb{J}_{\ell}\right\}$, i.e., $S$ is the sequence obtained by amalgamating $S_{1}, \ldots, S_{r}$. We write $S=\bigcup_{\ell=1}^{r} S_{\ell}$ for simplicity. $T=\bigcup_{\ell=1}^{r} T_{\ell}$ is defined similarly. Let $\ln S=\{\ln s: s \in S\}$. For any $s>0, t \in \mathbb{R}$ and $f \in L^{2}(\mathbb{R})$, define $\tau(s, t) f=s^{1 / 2} f(s \cdot-t)$.

With these symbols, the wavelet system $\left\{s_{\ell, j}^{1 / 2} \psi_{\ell}\left(s_{\ell, j} \cdot-t_{\ell, k}\right): j \in \mathbb{J}_{\ell}, k \in \mathbb{K}_{\ell}, 1 \leq\right.$ $\ell \leq r\}$ can be denoted by $\bigcup_{\ell=1}^{r}\left\{\tau(s, t) \psi_{\ell}: s \in S_{\ell}, t \in T_{\ell}\right\}$. We show that if a

Received by the editors February 3, 2003 and, in revised form, May 7, 2003.

2000 Mathematics Subject Classification. Primary 42C40, 41A58.

Key words and phrases. Wavelet frames, density, Beurling density.

This work was supported by the National Natural Science Foundation of China (10171050 and 10201014), the Mathematical Tianyuan Foundation (TY10126007), the Research Fund for the Doctoral Program of Higher Education, and the Liuhui Center for Applied Mathematics. 
wavelet system forms a frame for $L^{2}(\mathbb{R})$, then $\ln S$ and $T$ are relatively uniformly discrete, or equivalently, $\ln S$ and $T$ have finite upper Beurling densities. Moreover, we prove that if $\psi_{\ell}$ are admissible, then $\ln S$ possesses a positive lower Beurling density. We also give an example to show that the lower Beurling density of $T$ may be zero. Additionally, we prove that there are no frames generated by dilations of a finite number of admissible functions.

Notation and Definitions. The Fourier transform of $f \in L^{2}(\mathbb{R})$ is defined by $\hat{f}(\omega)=\int_{\mathbb{R}} f(x) e^{-i x \omega} d x$.

We call a function $\psi \in L^{2}(\mathbb{R})$ admissible if $C_{\psi}:=\int_{-\infty}^{+\infty} \frac{|\hat{\psi}(\omega)|^{2}}{|\omega|} d \omega<+\infty$.

$C_{c}(\mathbb{R})=\{f: f$ is continuous and compactly supported $\}$.

$\|f\|$ denotes the $L^{2}$-norm for any $f \in L^{2}(\mathbb{R})$.

$\# S$ denotes the number of elements in a set or a sequence $S$.

$\lfloor x\rfloor=\max \{n: n \leq x, n \in \mathbb{Z}\}$ and $\lceil x\rceil=\min \{n: n \geq x, n \in \mathbb{Z}\}$ for any $x \in \mathbb{R}$.

A sequence $\Gamma=\left\{\gamma_{i}: i \in I\right\} \subset \mathbb{R}$ is called $\delta$-uniformly discrete if $\left|\gamma_{i}-\gamma_{j}\right| \geq \delta>0$ for any $i, j \in I, i \neq j$. $\Gamma$ is called relatively uniformly discrete if it is a finite union of uniformly discrete sequences. The lower and upper Beurling densities of $\Gamma$ are defined respectively by

$$
\begin{aligned}
& D^{-}(\Gamma)=\liminf _{R \rightarrow+\infty} \frac{\min _{x \in \mathbb{R}} \#([x-R, x+R] \cap \Gamma)}{2 R}, \\
& D^{+}(\Gamma)=\limsup _{R \rightarrow+\infty} \frac{\max _{x \in \mathbb{R}} \#([x-R, x+R] \cap \Gamma)}{2 R} .
\end{aligned}
$$

It was shown in [4, Lemma 2.3] that a sequence is relatively uniformly discrete if and only if it has a finite upper Beurling density.

A family of functions $\left\{f_{j}: j \in \mathbb{J}\right\}$ belonging to a separable Hilbert space $\mathcal{H}$ is said to be a frame if there exist positive constants $A$ and $B$ such that $A\|f\|^{2} \leq$ $\sum_{j \in J}\left|\left\langle f, f_{j}\right\rangle\right|^{2} \leq B\|f\|^{2}$ for every $f \in \mathcal{H}$. The numbers $A$ and $B$ are called the lower and upper frame bounds, respectively.

Remark. After submitting this paper, we learned that Heil and Kutyniok [12] have simultaneously derived some interesting results on the density of weighted wavelet frames of the form $\left\{w(a, b)^{1 / 2} \tau(a, b) \psi:(a, b) \in \Lambda\right\}$, where $w(a, b)$ is a weight function and $\Lambda \subset \mathbb{R}^{+} \times \mathbb{R}$ is a sequence. However, their results are distinct from ours, and, in particular, we consider multi-generated wavelet systems. We also studied the density of wavelet frames with arbitrary sampling points in [19].

\section{MAin Results}

Theorem 2.1. Let $\psi_{\ell} \in L^{2}(\mathbb{R}), S_{\ell}$ and $T_{\ell}$ be real sequences, and let $S_{\ell}$ consist of positive numbers, $1 \leq \ell \leq r$. Denote $\bigcup_{\ell=1}^{r} S_{\ell}$ and $\bigcup_{\ell=1}^{r} T_{\ell}$ by $S$ and $T$, respectively.

(1) If $\bigcup_{\ell=1}^{r}\left\{\tau(s, t) \psi_{\ell}: s \in S_{\ell}, t \in T_{\ell}\right\}$ possesses a finite upper frame bound for $L^{2}(\mathbb{R})$, then both $\ln S$ and $T$ have finite upper Beauling densities, or equivalently, both are relatively uniformly discrete.

(2) If $\bigcup_{\ell=1}^{r}\left\{\tau(s, t) \psi_{\ell}: s \in S_{\ell}, t \in T_{\ell}\right\}$ is a frame for $L^{2}(\mathbb{R})$, then $\sup S=+\infty$. Moreover, if $\psi_{\ell}$ is admissible, $1 \leq \ell \leq r$, then

$$
\inf S=0, \inf T=-\infty, \quad \sup T=+\infty,
$$

and there is some constant $\Delta>1$ such that

$$
\#\left(\left[\Delta^{j}, \Delta^{j+1}\right] \cap S\right) \geq 1, \quad \forall j \in \mathbb{Z},
$$


which, in particular, implies that

$$
D^{-}(\ln S) \geq \frac{1}{\ln \Delta} .
$$

Corollary 2.2. Let $\psi_{\ell}, S_{\ell}$ and $T_{\ell}$ be defined as in Theorem [2.1. If $\bigcup_{\ell=1}^{r}\left\{\tau(s, t) \psi_{\ell}\right.$ : $\left.s \in S_{\ell}, t \in T_{\ell}\right\}$ is a frame for $L^{2}(\mathbb{R})$, then $\bigcup_{\ell=1}^{r} S_{\ell}$ is an infinite sequence.

Moreover, if $\psi_{\ell}$ is admissible, $1 \leq \ell \leq r$, then $\bigcup_{\ell=1}^{r} T_{\ell}$ is also an infinite sequence.

Remark. Olson and Zalik [16] proved that there does not exist any Riesz basis for $L^{2}(\mathbb{R})$ generated by translations of a single function. Moreover, Christensen, Deng and Heil 4 . proved that there are no frames for $L^{2}(\mathbb{R})$ generated by translations of finitely many functions, which coincides with the first part of Corollary 2.2 The corollary above also shows that if generating functions $\psi_{\ell}$ satisfy a very weak condition, i.e., they are admissible, then there are no frames for $L^{2}(\mathbb{R})$ generated by dilations of finitely many functions.

By (2.3), $\ln S$ possesses a positive lower Beurling density. So $S$ cannot be "too discrete" for $\bigcup_{\ell=1}^{r}\left\{\tau(s, t) \psi_{\ell}: s \in S_{\ell}, t \in T_{\ell}\right\}$ to be a frame. Does the same thing occur for $T$ ? The answer is, surprisingly, no! In fact, we have the following.

Theorem 2.3. Suppose that $\psi(x)$ is a nonzero, two times continuously differentiable and real-valued function, $x \psi(x), \psi^{\prime}(x), x \psi^{\prime}(x), x \psi^{\prime \prime}(x) \in L^{2}(\mathbb{R})$ and $\hat{\psi}(0)=0$. Then there are increasing sequences $\left\{s_{j}: j \in \mathbb{Z}\right\}$ and $\left\{t_{k}: k \in \mathbb{Z}\right\}$ such that $\left\{\tau\left(s_{j}, t_{k}\right) \psi: j, k \in \mathbb{Z}\right\}$ is a frame for $L^{2}(\mathbb{R})$, and

$$
D^{-}\left(\left\{t_{k}: k \in \mathbb{Z}\right\}\right)=0 .
$$

Remark. It is easy to see that (2.4) is equivalent to $\sup _{k \in \mathbb{Z}}\left(t_{k+1}-t_{k}\right)=+\infty$. By [14, Lemma 8], (2.4) implies that for $\left\{\tau\left(s_{j}, t_{k}\right) \psi: j, k \in \mathbb{Z}\right\}$ to be a frame, $\left\{e^{i t_{k} \omega}: k \in \mathbb{Z}\right\}$ may not be a frame for any $L^{2}[-r, r], r>0$. The following is an explicit example.

Example 2.1. Define $\hat{\psi}(\omega)=|\omega|^{\frac{1}{4}}(1-|\omega|)$ for $|\omega| \leq 1$ and 0 for others. Let $\Lambda=$ $\left\{k \in \mathbb{Z}: k \leq 2\right.$ or $2^{2 l} \leq k \leq 2^{2 l+1}$ for some $\left.l \geq 1\right\}$. Then $\left\{\tau\left(2^{j}, \frac{k}{2}\right) \psi: j \in \mathbb{Z}, k \in \Lambda\right\}$ is a frame for $L^{2}(\mathbb{R})$, and $D^{-}\left(\frac{1}{2} \Lambda\right)=0$.

\section{Proofs of THEOREMS}

We need only to prove Theorems 2.1 and 2.3, since Corollary 2.2 is an obvious consequence of Theorem 2.1.

Proof of Theorem 2.1(1). Let $B$ be the upper frame bound. We will prove that $\ln S$ and $T$ are relatively uniformly discrete. It suffices to show that $\ln S_{\ell}$ and $T_{\ell}$ are relatively uniformly discrete for any $1 \leq \ell \leq r$.

Fix some $1 \leq \ell \leq r, s_{0} \in S_{\ell}$, and $t_{0} \in T_{\ell}$. Let $f=\alpha \tau\left(s_{0}, t_{0}\right) \psi_{\ell}$, where the constant $\alpha$ is chosen so that $\|f\|=1$. Then

$$
c:=\left|\left\langle f, \tau\left(s_{0}, t_{0}\right) \psi_{\ell}\right\rangle\right|^{2}>0 .
$$

It is easy to check that $\left\langle f, \tau(s, t) \psi_{\ell}\right\rangle$ is continuous with respect to $s$ and $t$. Hence there is some $a_{\ell}>1$ such that

$$
\left|\left\langle f, \tau\left(s s_{0}, t_{0}\right) \psi_{\ell}\right\rangle\right|^{2}>\frac{c}{2}, \quad \forall a_{\ell}^{-1}<s<a_{\ell} .
$$


For any $j \in \mathbb{Z}$, we have

$$
\begin{aligned}
B & =B\left\|\tau\left(a_{\ell}^{j} / s_{0}, 0\right) f\right\|^{2} \geq \sum_{s \in S_{\ell}, t \in T_{\ell}}\left|\left\langle\tau\left(a_{\ell}^{j} / s_{0}, 0\right) f, \tau(s, t) \psi_{\ell}\right\rangle\right|^{2} \\
& \geq \sum_{s \in S_{\ell}}\left|\left\langle\tau\left(a_{\ell}^{j} / s_{0}, 0\right) f, \tau\left(s, t_{0}\right) \psi_{\ell}\right\rangle\right|^{2}=\sum_{s \in S_{\ell}}\left|\left\langle f, \tau\left(s s_{0} / a_{\ell}^{j}, t_{0}\right) \psi_{\ell}\right\rangle\right|^{2} .
\end{aligned}
$$

It follows from (3.1) that $\#\left\{s \in S_{\ell}: \frac{1}{a_{\ell}}<\frac{s}{a_{\ell}^{j}}<a_{\ell}\right\} \leq p:=\left\lfloor\frac{2 B}{c}\right\rfloor$. Hence there are at most $p$ elements of $S_{\ell}$ in each interval $\left[a_{\ell}^{j}, a_{\ell}^{j+1}\right), j \in \mathbb{Z}$. Therefore, we can split $S_{\ell}$ into at most $2 p$ subsequences $S_{\ell, n}, 1 \leq n \leq 2 p$, such that $S_{\ell, 2 n-1} \subset$ $\bigcup_{j \in \mathbb{Z}}\left[a_{\ell}^{2 j-1}, a_{\ell}^{2 j}\right), S_{\ell, 2 n} \subset \bigcup_{j \in \mathbb{Z}}\left[a_{\ell}^{2 j}, a_{\ell}^{2 j+1}\right)$, and $\#\left(S_{\ell, n} \cap\left[a_{\ell}^{j}, a_{\ell}^{j+1}\right)\right) \leq 1, \forall j \in \mathbb{Z}$. It follows that

$$
\left|\ln s-\ln s^{\prime}\right| \geq \ln a_{\ell}, \quad \forall s, s^{\prime} \in S_{\ell, n}, s \neq s^{\prime} .
$$

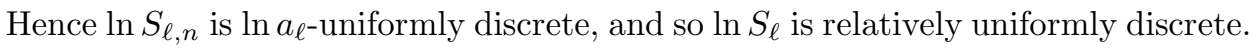

Next we will prove that $T_{\ell}$ is relatively uniformly discrete. By continuity, there is some $b_{\ell}>0$ such that

$$
\left|\left\langle f, \tau\left(s_{0}, t_{0}+t\right) \psi_{\ell}\right\rangle\right|^{2}>\frac{c}{2}, \quad|t|<b_{\ell}
$$

For any $k \in \mathbb{Z}$, let $x_{k} \in \mathbb{R}$ be such that $s_{0} x_{k}+k b_{\ell}=t_{0}$. We have

$$
\begin{aligned}
B & =B\left\|f\left(\cdot+x_{k}\right)\right\|^{2} \geq \sum_{s \in S_{\ell}, t \in T_{\ell}}\left|\left\langle f\left(\cdot+x_{k}\right), \tau(s, t) \psi_{\ell}\right\rangle\right|^{2} \\
& \geq \sum_{t \in T_{\ell}}\left|\left\langle f\left(\cdot+x_{k}\right), \tau\left(s_{0}, t\right) \psi_{\ell}\right\rangle\right|^{2}=\sum_{t \in T_{\ell}}\left|\left\langle f, \tau\left(s_{0}, s_{0} x_{k}+t\right) \psi_{\ell}\right\rangle\right|^{2} \\
& =\sum_{t \in T_{\ell}}\left|\left\langle f, \tau\left(s_{0}, t_{0}+\left(t-k b_{\ell}\right)\right) \psi_{\ell}\right\rangle\right|^{2} .
\end{aligned}
$$

By (3.2), we have $\#\left\{t \in T_{\ell}:\left|t-k b_{\ell}\right|<b_{\ell}\right\} \leq q:=\left\lfloor\frac{2 B}{c}\right\rfloor$. Hence we can split $T_{\ell}$ into at most $2 q$ subsequences $T_{\ell, m}, 1 \leq m \leq 2 q$ such that

$$
T_{\ell, 2 m-1} \subset \bigcup_{k \in \mathbb{Z}}\left[(2 k-1) b_{\ell}, 2 k b_{\ell}\right), \quad T_{\ell, 2 m} \subset \bigcup_{k \in \mathbb{Z}}\left[2 k b_{\ell},(2 k+1) b_{\ell}\right),
$$

and

$$
\#\left(T_{\ell, m} \bigcap\left[k b_{\ell},(k+1) b_{\ell}\right)\right) \leq 1, \quad \forall k \in \mathbb{Z} .
$$

Hence $T_{\ell}$ is relatively uniformly discrete.

Before proving Theorem 2.1 (2), we introduce some preliminary results.

Lemma 3.1. For any $b>0$, there is some constant $C_{b}>0$ such that for any $c<d$, any $f \in L^{2}[c, d]$, and any b-uniformly discrete sequence $\left\{t_{k}: k \in \mathbb{Z}\right\}$,

$$
\sum_{k \in \mathbb{Z}}\left|\int_{c}^{d} f(x) e^{i t_{k} x} d x\right|^{2} \leq 4 \pi^{2}\lceil d-c\rceil C_{b} \int_{c}^{d}|f(x)|^{2} d x .
$$

Proof. By 1, Lemma 42], there is some constant $C_{b}$ such that for any $b$-uniformly discrete sequence $\left\{t_{k}: k \in \mathbb{Z}\right\}$ and $f \in L^{2}[0,1]$,

$$
\sum_{k \in \mathbb{Z}}\left|\int_{0}^{1} f(x) e^{i t_{k} x} d x\right|^{2} \leq 4 \pi^{2} C_{b} \int_{0}^{1}|f(x)|^{2} d x .
$$


If $d-c \leq 1$, then (3.3) follows by a change of variable of the form $x \rightarrow x+c$. For the case of $d-c>1$, let $n=\lceil d-c\rceil$. Then $\Delta:=(d-c) / n \leq 1$. Hence

$$
\begin{aligned}
& \sum_{k \in \mathbb{Z}}\left|\int_{c}^{d} f(x) e^{i t_{k} x} d x\right|^{2}=\sum_{k \in \mathbb{Z}}\left|\sum_{l=0}^{n-1} \int_{c+l \Delta}^{c+(l+1) \Delta} f(x) e^{i t_{k} x} d x\right|^{2} \\
\leq & \sum_{k \in \mathbb{Z}} n \sum_{l=0}^{n-1}\left|\int_{c+l \Delta}^{c+(l+1) \Delta} f(x) e^{i t_{k} x} d x\right|^{2} \leq n \sum_{l=0}^{n-1} 4 \pi^{2} C_{b} \int_{c+l \Delta}^{c+(l+1) \Delta}|f(x)|^{2} d x \\
= & \lceil d-c\rceil \cdot 4 \pi^{2} C_{b} \int_{c}^{d}|f(x)|^{2} d x .
\end{aligned}
$$

Lemma 3.2. Let $\psi \in L^{2}(\mathbb{R})$, let $a>1, b>0$ be constants, and let $C_{b}$ be defined as in Lemma 3.1. Let $f \in L^{2}(\mathbb{R})$ be such that $\operatorname{supp} \hat{f} \subset[1, a]$ and $\|\hat{f}\|_{\infty}<\infty$. Then

$$
\begin{aligned}
\sum_{s_{j}>M, k \in \mathbb{Z}}\left|\left\langle f, \tau\left(s_{j}, t_{j, k}\right) \psi\right\rangle\right|^{2} & \leq a C_{b}\|\hat{f}\|_{\infty}^{2} \int_{0}^{\frac{a}{M}} \frac{|\hat{\psi}(\omega)|^{2}}{\omega} d \omega \\
\sum_{s_{j}<\frac{1}{M}, k \in \mathbb{Z}}\left|\left\langle f, \tau\left(s_{j}, t_{j, k}\right) \psi\right\rangle\right|^{2} & \leq 2(a-1) C_{b}\|\hat{f}\|_{\infty}^{2} \int_{M}^{+\infty}|\hat{\psi}(\omega)|^{2} d \omega
\end{aligned}
$$

whenever $\left\{\ln s_{j}: j \in \mathbb{Z}\right\}$ is $\ln$ a-uniformly discrete, $\left\{t_{j, k}: k \in \mathbb{Z}\right\}$ is b-uniformly discrete, $M>a-1$ for (3.4) and $M>\frac{1}{a-1}$ for (3.5).

Proof. Since supp $\hat{f} \subset[1, a]$ and $\frac{a}{s_{j}}-\frac{1}{s_{j}} \leq \frac{a-1}{M}<1$ for any $s_{j}>M>a-1$, we derive from Lemma 3.1 that

$$
\begin{aligned}
& \sum_{s_{j}>M, k \in \mathbb{Z}}\left|\left\langle f, \tau\left(s_{j}, t_{j, k}\right) \psi\right\rangle\right|^{2}=\sum_{s_{j}>M, k \in \mathbb{Z}}\left|\frac{1}{2 \pi} \int_{-\infty}^{+\infty} \hat{f}(\omega) s_{j}^{-\frac{1}{2}} \overline{\hat{\psi}\left(\frac{\omega}{s_{j}}\right)} e^{i t_{j, k} s_{j}^{-1} \omega} d \omega\right|^{2} \\
= & \sum_{s_{j}>M, k \in \mathbb{Z}}\left|\frac{1}{2 \pi} \int_{\frac{1}{s_{j}}}^{\frac{a}{s_{j}}} s_{j}^{\frac{1}{2}} \hat{f}\left(s_{j} \omega\right) \overline{\hat{\psi}(\omega)} e^{i t_{j, k} \omega} d \omega\right|^{2} \\
\leq & \sum_{s_{j}>M} C_{b} \int_{\frac{1}{s_{j}}}^{\frac{a}{s_{j}}}\left|s_{j}^{\frac{1}{2}} \hat{f}\left(s_{j} \omega\right) \hat{\psi}(\omega)\right|^{2} d \omega \leq \sum_{s_{j}>M} C_{b} \int_{\frac{1}{s_{j}}}^{\frac{a}{s_{j}}} \frac{a}{\omega}\|\hat{f}\|_{\infty}^{2}|\hat{\psi}(\omega)|^{2} d \omega .
\end{aligned}
$$

Since $\left\{\ln s_{j}: j \in \mathbb{Z}\right\}$ is $\ln a$-uniformly discrete, the intervals $\left[\frac{1}{s_{j}}, \frac{a}{s_{j}}\right)$ are mutually disjoint. Hence (3.4) holds. have

Noting that $\left\lceil\frac{a-1}{s_{j}}\right\rceil \leq \frac{2(a-1)}{s_{j}}$ for any $s_{j}<\frac{1}{M}<a-1$, using Lemma 3.1]again, we

$$
\begin{aligned}
& \sum_{s_{j}<\frac{1}{M}, k \in \mathbb{Z}}\left|\left\langle f, \tau\left(s_{j}, t_{j, k}\right) \psi\right\rangle\right|^{2}=\sum_{s_{j}<\frac{1}{M}, k \in \mathbb{Z}}\left|\frac{1}{2 \pi} \int_{\frac{1}{s_{j}}}^{\frac{a}{s_{j}}} s_{j}^{1 / 2} \hat{f}\left(s_{j} \omega\right) \overline{\hat{\psi}(\omega)} e^{i t_{j, k} \omega} d \omega\right|^{2} \\
\leq & \sum_{s_{j}<\frac{1}{M}} \frac{2(a-1)}{s_{j}} C_{b} \int_{\frac{1}{s_{j}}}^{\frac{a}{s_{j}}}\left|s_{j}^{1 / 2} \hat{f}\left(s_{j} \omega\right) \hat{\psi}(\omega)\right|^{2} d \omega \\
\leq & \sum_{s_{j}<\frac{1}{M}} 2(a-1) C_{b} \int_{\frac{1}{s_{j}}}^{\frac{a}{s_{j}}}\|\hat{f}\|_{\infty}^{2}|\hat{\psi}(\omega)|^{2} d \omega \leq 2(a-1) C_{b}\|\hat{f}\|_{\infty}^{2} \int_{M}^{+\infty}|\hat{\psi}(\omega)|^{2} d \omega .
\end{aligned}
$$

This completes the proof. 
For any $f, \psi \in L^{2}(\mathbb{R})$, define

$$
\left(T_{\psi} f\right)(s, t)=\left\langle f,|s|^{1 / 2} \psi(s \cdot-t)\right\rangle, \quad s, t \in \mathbb{R} .
$$

Since $\left(T_{\psi} f\right)(s, t)=\left\langle|s|^{-\frac{1}{2}} f\left(\frac{\cdot+t}{s}\right), \psi\right\rangle$ for any $s \neq 0$, we see from wavelet theory that if $f$ is admissible, then

$$
\iint_{\mathbb{R}^{2}} \frac{1}{s^{2}}\left|\left(T_{\psi} f\right)(s, t)\right|^{2} d t d s=C_{f}\|\psi\|^{2}<+\infty .
$$

The following lemma is a consequence of the Wirtinger inequality [11].

Lemma 3.3. If $f(x)$ is differentiable on $[a, b], f, f^{\prime} \in L^{2}[a, b]$ and there is some $c \in[a, b]$ such that $f(c)=0$, then $\int_{a}^{b}|f(x)|^{2} d x \leq\left(\frac{2 \max \{c-a, b-c\}}{\pi}\right)^{2} \int_{a}^{b}\left|f^{\prime}(x)\right|^{2} d x$.

Lemma 3.4. Suppose that $\hat{f}(\omega)$ is compactly supported and continuously differentiable and that $\hat{f}(0)=0$. For any $\psi \in L^{2}(\mathbb{R}), \varepsilon, b>0$ and $M, a>1$, there is some constant $N=N(\varepsilon, M, a, b, \psi, f)>0$ such that

$$
\sum_{s_{j} \in\left[\frac{1}{M}, M\right]} \sum_{\left|t_{j, k}\right|>N}\left|\left\langle f, s_{j}^{1 / 2} \psi\left(s_{j} \cdot-t_{j, k}\right)\right\rangle\right|^{2}<\varepsilon
$$

for any $s_{j}$ and $t_{j, k}$ for which $\left\{\ln s_{j}: j \in \mathbb{Z}\right\}$ is $\ln$ a-uniformly discrete and $\left\{t_{j, k}\right.$ : $k \in \mathbb{Z}\}$ is b-uniformly discrete.

Proof. Since $\hat{f}$ is compactly supported, $f \in C^{\infty}$. Noting that

$$
\left(T_{\psi} f\right)(s, t)=\left\langle s^{-\frac{1}{2}} f\left(\frac{\cdot+t}{s}\right), \psi\right\rangle,
$$

we have

$$
\frac{\partial}{\partial t}\left(T_{\psi} f\right)(s, t)=\frac{1}{s}\left(T_{\psi} f^{\prime}\right)(s, t), \quad \frac{\partial}{\partial s} \frac{1}{s}\left(T_{\psi} f\right)(s, t)=-\frac{1}{s^{2}}\left(T_{\psi} \tilde{f}\right)(s, t), \quad s>0, t \in \mathbb{R},
$$

where $\tilde{f}(x)=\frac{3}{2} f(x)+x f^{\prime}(x)$.

For any $j, k \in \mathbb{Z}$, let $Q_{j, k}=\left[s_{j} a^{-1 / 2}, s_{j} a^{1 / 2}\right] \times\left[t_{j, k}-\frac{s_{j} b}{2 M}, t_{j, k}+\frac{s_{j} b}{2 M}\right]$. Then the Lebesgue measure of $Q_{j, k} \cap Q_{j^{\prime}, k^{\prime}}$ equals zero whenever $(j, k) \neq\left(j^{\prime}, k^{\prime}\right)$ and $s_{j}, s_{j^{\prime}} \leq M$. It follows from Lemma 3.3 that for any $N>0$,

$$
\begin{aligned}
& \sum_{s_{j} \in\left[\frac{1}{M}, M\right]} \sum_{\left|t_{j, k}\right|>N} \iint_{Q_{j, k}} \frac{1}{s^{2}}\left|\left(T_{\psi} f\right)(s, t)-\left(T_{\psi} f\right)\left(s, t_{j, k}\right)\right|^{2} d t d s \\
= & \sum_{s_{j} \in\left[\frac{1}{M}, M\right]} \sum_{\left|t_{j, k}\right|>N} \int_{s_{j} a^{-\frac{1}{2}}}^{s_{j} a^{\frac{1}{2}}} \frac{d s}{s^{2}} \int_{t_{j, k}-\frac{s_{j} b}{2 M}}^{t_{j, k}+\frac{s_{j} b}{2 M}}\left|\left(T_{\psi} f\right)(s, t)-\left(T_{\psi} f\right)\left(s, t_{j, k}\right)\right|^{2} d t \\
\leq & \sum_{s_{j} \in\left[\frac{1}{M}, M\right]} \sum_{\left|t_{j, k}\right|>N} \int_{s_{j} a^{-\frac{1}{2}}}^{s_{j} a^{\frac{1}{2}}} \frac{d s}{s^{2}} \cdot \frac{s_{j}^{2} b^{2}}{\pi^{2} M^{2}} \int_{t_{j, k}-\frac{s_{j} b}{2 M}}^{t_{j, k}+\frac{s_{j} b}{2 M}} \frac{1}{s^{2}}\left|\left(T_{\psi} f^{\prime}\right)(s, t)\right|^{2} d t \\
\leq & \sum_{s_{j} \in\left[\frac{1}{M}, M\right]} \sum_{\left|t_{j, k}\right|>N} \frac{a b^{2}}{\pi^{2} M^{2}} \iint_{Q_{j, k}} \frac{1}{s^{2}}\left|\left(T_{\psi} f^{\prime}\right)(s, t)\right|^{2} d t d s \\
\leq & \frac{a b^{2}}{\pi^{2} M^{2}} \int_{s \in\left[\frac{1}{M \sqrt{a}}, M \sqrt{a}\right],|t|>N-b} \frac{1}{s^{2}}\left|\left(T_{\psi} f^{\prime}\right)(s, t)\right|^{2} d t d s .
\end{aligned}
$$


Hence,

$$
\begin{aligned}
& \sum_{s_{j} \in\left[\frac{1}{M}, M\right]} \sum_{\left|t_{j, k}\right|>N} \iint_{Q_{j, k}} \frac{1}{s^{2}}\left|\left(T_{\psi} f\right)\left(s, t_{j, k}\right)\right|^{2} d t d s \\
\leq & \sum_{s_{j} \in\left[\frac{1}{M}, M\right]} \sum_{\left|t_{j, k}\right|>N} \iint_{Q_{j, k}} \frac{2}{s^{2}}\left(\left|\left(T_{\psi} f\right)(s, t)\right|^{2}+\left|\left(T_{\psi} f\right)(s, t)-\left(T_{\psi} f\right)\left(s, t_{j, k}\right)\right|^{2}\right) d t d s \\
\leq & \iint_{s \in\left[\frac{1}{M \sqrt{a}}, M \sqrt{a}\right],|t|>N-b}\left(\frac{2}{s^{2}}\left|\left(T_{\psi} f\right)(s, t)\right|^{2}+\frac{a b^{2}}{\pi^{2} M^{2}} \cdot \frac{2}{s^{2}}\left|\left(T_{\psi} f^{\prime}\right)(s, t)\right|^{2}\right) d t d s \\
:= & P .
\end{aligned}
$$

Therefore,

$$
\begin{aligned}
& \sum_{s_{j} \in\left[\frac{1}{M}, M\right]} \sum_{\left|t_{j, k}\right|>N} \iint_{Q_{j, k}}\left|\frac{1}{s}\left(T_{\psi} f\right)\left(s, t_{j, k}\right)-\frac{1}{s_{j}}\left(T_{\psi} f\right)\left(s_{j}, t_{j, k}\right)\right|^{2} d t d s \\
= & \sum_{s_{j} \in\left[\frac{1}{M}, M\right]} \sum_{\left|t_{j, k}\right|>N} \int_{t_{j, k}-\frac{s_{j} b}{2 M}}^{t_{j, k}+\frac{s_{j} b}{2 M}} d t \int_{s_{j} a^{-\frac{1}{2}}}^{s_{j} a^{\frac{1}{2}}}\left|\frac{1}{s}\left(T_{\psi} f\right)\left(s, t_{j, k}\right)-\frac{1}{s_{j}}\left(T_{\psi} f\right)\left(s_{j}, t_{j, k}\right)\right|^{2} d s
\end{aligned}
$$$$
\leq \sum_{s_{j} \in\left[\frac{1}{M}, M\right]} \sum_{\left|t_{j, k}\right|>N} \frac{4 s_{j}^{2}(\sqrt{a}-1)^{2}}{\pi^{2}} \iint_{Q_{j, k}} \frac{1}{s^{4}}\left|\left(T_{\psi} \tilde{f}\right)\left(s, t_{j, k}\right)\right|^{2} d t d s
$$$$
\leq \sum_{s_{j} \in\left[\frac{1}{M}, M\right]} \sum_{\left|t_{j, k}\right|>N} \frac{4 a(\sqrt{a}-1)^{2}}{\pi^{2}} \iint_{Q_{j, k}} \frac{1}{s^{2}}\left|\left(T_{\psi} \tilde{f}\right)\left(s, t_{j, k}\right)\right|^{2} d t d s
$$$$
\leq \quad \iint_{\substack{s \in\left[\frac{1}{M \sqrt{a}}, M \sqrt{a}\right] \\|t|>N-b}} \frac{8 a(\sqrt{a}-1)^{2}}{\pi^{2}} \cdot \frac{1}{s^{2}}\left(\left|\left(T_{\psi} \tilde{f}\right)(s, t)\right|^{2}+\frac{a b^{2}}{\pi^{2} M^{2}}\left|\left(T_{\psi}(\tilde{f})^{\prime}\right)(s, t)\right|^{2}\right) d t d s
$$

$:=Q$,

where (3.7) is used in the last inequality. Putting (3.7) and (3.8) together, we get

$$
\begin{aligned}
& \sum_{\substack{s_{j} \in\left[\frac{1}{M}, M\right] \\
\left|t_{j, k}\right|>N}}\left|\left\langle f, \tau\left(s_{j}, t_{j, k}\right) \psi\right\rangle\right|^{2}=\frac{M \sqrt{a}}{b(a-1)} \sum_{\substack{s_{j} \in\left[\frac{1}{M}, M\right] \\
\left|t_{j, k}\right|>N}} \iint_{Q_{j, k}} \frac{1}{s_{j}^{2}}\left|\left(T_{\psi} f\right)\left(s_{j}, t_{j, k}\right)\right|^{2} d t d s \\
= & \frac{M \sqrt{a}}{b(a-1)} \sum_{\substack{s_{j} \in\left[\frac{1}{M}, M\right] \\
\left|t_{j, k}\right|>N}} \iint\left|\frac{1}{Q_{j, k}}\left(T_{\psi} f\right)\left(s, t_{j, k}\right)-\left(\frac{1}{s}\left(T_{\psi} f\right)\left(s, t_{j, k}\right)-\frac{1}{s_{j}}\left(T_{\psi} f\right)\left(s_{j}, t_{j, k}\right)\right)\right|^{2} d t d s \\
\leq & \frac{M \sqrt{a}}{b(a-1)}(2 P+2 Q) .
\end{aligned}
$$

Since $\hat{f}$ is continuously differentiable and $\hat{f}(0)=0$, it is easy to check that all of $f, f^{\prime}, \tilde{f}$, and $(\tilde{f})^{\prime}$ are admissible. By (3.6), all of $\frac{1}{s}\left(T_{\psi} f\right)(s, t), \frac{1}{s}\left(T_{\psi} f^{\prime}\right)(s, t)$, $\frac{1}{s}\left(T_{\psi} \tilde{f}\right)(s, t)$, and $\frac{1}{s}\left(T_{\psi}(\tilde{f})^{\prime}\right)(s, t)$ are square integrable on $\mathbb{R}^{2}$. Hence

$$
\lim _{N \rightarrow+\infty}(P+Q)=0
$$

which completes the proof. 
Proof of Theorem $2.1(2)$. Let $A$ and $B$ be the lower and upper frame bounds, respectively. By (i), there exist constants $a_{\ell}>1, b_{\ell}>0$ and positive integers $p$ and $q$ such that $S_{\ell}=\bigcup_{n=1}^{2 p} S_{\ell, n}, T_{\ell}=\bigcup_{m=1}^{2 q} T_{\ell, m}, \ln S_{\ell, n}$ is $\ln a_{\ell}$-uniformly discrete and $T_{\ell, m}$ is $b_{\ell}$-uniformly discrete. Let $a=\min _{1 \leq \ell \leq r} a_{\ell}$ and $b=\min _{1 \leq \ell \leq r} b_{\ell}$. Then $\ln S_{\ell, n}$ is $\ln a$-uniformly discrete and $T_{\ell, m}$ is $b$-uniformly discrete for any $1 \leq \ell \leq r$, $1 \leq n \leq 2 p$, and $1 \leq m \leq 2 q$.

Put $\hat{f}(\omega)=\frac{1}{\sqrt{a-1}} \chi_{[1, a]}(\omega)$. If $\alpha=\sup S<+\infty$, then (3.5) implies that for $\delta$ small enough,

$$
\sum_{s \in S_{\ell, n}, t \in T_{\ell, m}}\left|\left\langle f, \tau(s \delta, t) \psi_{\ell}\right\rangle\right|^{2} \leq 2 C_{b} \int_{\frac{1}{\delta \alpha}}^{+\infty}\left|\hat{\psi}_{\ell}(\omega)\right|^{2} d \omega, \quad \forall \ell, n, m .
$$

Hence

$$
\begin{gathered}
A\|f\|^{2}=A\|\tau(1 / \delta, 0) f\|^{2} \leq \sum_{\ell=1}^{r} \sum_{s \in S_{\ell}, t \in T_{\ell}}\left|\left\langle\tau\left(\frac{1}{\delta}, 0\right) f, \tau(s, t) \psi_{\ell}\right\rangle\right|^{2} \\
=\sum_{\ell=1}^{r} \sum_{n=1}^{2 p} \sum_{m=1}^{2 q} \sum_{s \in S_{\ell, n}, t \in T_{\ell, m}}\left|\left\langle f, \tau(s \delta, t) \psi_{\ell}\right\rangle\right|^{2} \leq 8 p q C_{b} \sum_{\ell=1}^{r} \int_{\frac{1}{\delta \alpha}}^{+\infty}\left|\hat{\psi}_{\ell}(\omega)\right|^{2} d \omega .
\end{gathered}
$$

By letting $\delta \rightarrow 0$, we get $A=0$, which is impossible. Hence $\sup S=+\infty$.

In what follows we assume that $\psi_{\ell}$ is admissible, $1 \leq \ell \leq r$.

Assume that $\beta=\inf S>0$. By (3.4), for any $0<\delta<\frac{\beta}{a-1}$, we have

$$
\begin{aligned}
& A\|f\|^{2}=A\|\tau(\delta, 0) f\|^{2} \leq \sum_{\ell=1}^{r} \sum_{s \in S_{\ell}, t \in T_{\ell}}\left|\left\langle\tau(\delta, 0) f, \tau(s, t) \psi_{\ell}\right\rangle\right|^{2} \\
= & \sum_{\ell=1}^{r} \sum_{n=1}^{2 p} \sum_{m=1}^{2 q} \sum_{s \in S_{\ell, n}, t \in T_{\ell, m}}\left|\left\langle f, \tau(s / \delta, t) \psi_{\ell}\right\rangle\right|^{2} \leq 4 p q \cdot \frac{a C_{b}}{a-1} \sum_{\ell=1}^{r} \int_{0}^{\frac{\delta a}{\beta}} \frac{\left|\hat{\psi}_{\ell}(\omega)\right|^{2}}{|\omega|} d \omega .
\end{aligned}
$$

By letting $\delta \rightarrow 0$ we get $A=0$, which is a contradiction. Hence inf $S=0$.

Next we will prove that $\inf T=-\infty$. Choose some $f \neq 0$ such that $\hat{f}$ is continuously differentiable and supp $\hat{f} \subset[1, a]$. Since $\ln S_{\ell, n}$ is $\ln a$-uniformly discrete and $s x+T_{\ell, m}:=\left\{s x+t: t \in T_{\ell, m}\right\}$ is $b$-uniformly discrete for any $\ell, n, m$ and $s x$, it follows from Lemma 3.2 that for any $M>\max \left\{a-1, \frac{1}{a-1}\right\}$,

$$
\begin{aligned}
& \sum_{s \in S_{\ell, n} \backslash\left\{\frac{1}{M}, M\right]} \sum_{t \in T_{\ell, m}}\left|\left\langle f, \tau(s, s x+t) \psi_{\ell}\right\rangle\right|^{2} \\
\leq & a C_{b}\|\hat{f}\|_{\infty}^{2} \int_{0}^{\frac{a}{M}} \frac{\left|\hat{\psi}_{\ell}(\omega)\right|^{2}}{\omega} d \omega+2(a-1) C_{b}\|\hat{f}\|_{\infty}^{2} \int_{M}^{+\infty}\left|\hat{\psi}_{\ell}(\omega)\right|^{2} d \omega .
\end{aligned}
$$

Hence, there is some $M>1$ such that

$$
\sum_{s \in S_{\ell, n} \backslash\left[\frac{1}{M}, M\right]} \sum_{t \in T_{\ell, m}}\left|\left\langle f, \tau(s, s x+t) \psi_{\ell}\right\rangle\right|^{2}<\frac{1}{4 p q r} \cdot \frac{A}{2}\|f\|^{2}, \quad \forall x, l, m, n .
$$

Therefore,

$$
\sum_{\ell=1}^{r} \sum_{s \in S_{\ell} \backslash\left[\frac{1}{M}, M\right]} \sum_{t \in T_{\ell}}\left|\left\langle f, \tau(s, s x+t) \psi_{\ell}\right\rangle\right|^{2}<\frac{A}{2}\|f\|^{2}, \quad \forall x \in \mathbb{R} .
$$


But

$$
\sum_{\ell=1}^{r} \sum_{s \in S_{\ell}} \sum_{t \in T_{\ell}}\left|\left\langle f, \tau(s, s x+t) \psi_{\ell}\right\rangle\right|^{2}=\sum_{\ell=1}^{r} \sum_{s \in S_{\ell}} \sum_{t \in T_{\ell}}\left|\left\langle f(\cdot+x), \tau(s, t) \psi_{\ell}\right\rangle\right|^{2} \geq A\|f\|^{2} .
$$

Hence,

$$
\sum_{\ell=1}^{r} \sum_{s \in S_{\ell} \cap\left[\frac{1}{M}, M\right]} \sum_{t \in T_{\ell}}\left|\left\langle f, \tau(s, s x+t) \psi_{\ell}\right\rangle\right|^{2}>\frac{A}{2}\|f\|^{2}, \quad \forall x \in \mathbb{R} .
$$

On the other hand, by Lemma 3.4, there is some $N>0$ such that

$$
\sum_{s \in S_{\ell, n} \cap\left[\frac{1}{M}, M\right]} \sum_{t \in T_{\ell, n} \cap\{|s x+t|>N\}}\left|\left\langle f, \tau(s, s x+t) \psi_{\ell}\right\rangle\right|^{2}<\frac{1}{4 p q r} \cdot \frac{A}{2}\|f\|^{2}, \quad \forall x, \ell, m, n .
$$

Hence,

$$
\sum_{\ell=1}^{r} \sum_{s \in S_{\ell} \cap\left[\frac{1}{M}, M\right]} \sum_{t \in T_{\ell} \cap\{|s x+t|>N\}}\left|\left\langle f, \tau(s, s x+t) \psi_{\ell}\right\rangle\right|^{2}<\frac{A}{2}\|f\|^{2}, \quad \forall x .
$$

If $t_{0}:=\inf T>-\infty$, then there is some $x_{0}>0$ such that $\frac{x_{0}}{M}+t_{0}>N$. Thus $s x_{0}+t \geq \frac{x_{0}}{M}+t_{0}>N$ for any $s \geq \frac{1}{M}$ and $t \in T$. By setting $x=x_{0}$ in the inequality above, we have

$$
\sum_{\ell=1}^{r} \sum_{s \in S_{\ell} \cap\left[\frac{1}{M}, M\right]} \sum_{t \in T_{\ell}}\left|\left\langle f, \tau\left(s, s x_{0}+t\right) \psi_{\ell}\right\rangle\right|^{2}<\frac{A}{2}\|f\|^{2},
$$

which contradicts (3.9). Similarly we can prove that $\sup T=+\infty$.

At last, we will prove (2.2). We argue by contradiction and assume that for any $\Delta>1$, there is some $j_{0} \in \mathbb{Z}$ such that $\#\left(S \cap\left[\Delta^{j_{0}}, \Delta^{j_{0}+1}\right]\right)=0$. Let $\alpha=\Delta^{j_{0}+1 / 2}$. Then $\frac{s}{\alpha}>\Delta^{1 / 2}$ or $\frac{s}{\alpha}<\Delta^{-1 / 2}$ for any $s \in S$.

Let $\hat{f}(\omega)=\frac{1}{\sqrt{a-1}} \chi_{[1, a]}(\omega)$. It follows from Lemma 3.2 that for $\Delta$ large enough,

$$
\begin{aligned}
& A\|f\|^{2} \leq \sum_{\ell=1}^{r} \sum_{\substack{s \in S_{\ell} \\
t \in T_{\ell}}}\left|\left\langle\tau(\alpha, 0) f, \tau(s, t) \psi_{\ell}\right\rangle\right|^{2}=\sum_{\ell=1}^{r} \sum_{\substack{1 \leq n \leq 2 p \\
1 \leq m \leq 2 q}} \sum_{\substack{s \in S_{\ell, n} \\
t \in T_{\ell, m}}}\left|\left\langle f, \tau\left(\frac{s}{\alpha}, t\right) \psi_{\ell}\right\rangle\right|^{2} \\
\leq & \sum_{\ell=1}^{r} 4 p q\left(\frac{a C_{b}}{a-1} \int_{0}^{\frac{a}{\Delta^{1 / 2}}} \frac{\left|\hat{\psi}_{\ell}(\omega)\right|^{2}}{|\omega|} d \omega+2 C_{b} \int_{\Delta^{1 / 2}}^{+\infty}\left|\hat{\psi}_{\ell}(\omega)\right|^{2} d \omega\right) .
\end{aligned}
$$

By letting $\Delta \rightarrow+\infty$, we get $A=0$, which contradicts the hypotheses.

Lemma 3.5 ([18, Theorem 2.4]). Let $\psi$ be defined as in Theorem [2.3. Let a> $1, b>0$ be constants such that

$$
\Delta:=\frac{2 b}{\pi} C_{\psi^{\prime}}^{\frac{1}{2}}+\frac{2(a-1)}{\pi} C_{\tilde{\psi}}^{\frac{1}{2}}+\frac{4 b(a-1)}{\pi^{2}} C_{(\tilde{\psi})^{\prime}}^{\frac{1}{2}}<C_{\psi}^{\frac{1}{2}},
$$

where $\tilde{\psi}(x)=\frac{3}{2} \psi(x)+x \psi^{\prime}(x)$. Then $\left\{\tau\left(s_{j, k}, t_{j, k}\right) \psi: j, k \in \mathbb{Z}\right\}$ is a frame for $L^{2}(\mathbb{R})$ with bounds $\frac{1}{2 b(a-1)}\left(C_{\psi}^{\frac{1}{2}}-\Delta\right)^{2}$ and $\frac{a^{2}}{2 b(a-1)}\left(C_{\psi}^{\frac{1}{2}}+\Delta\right)^{2}$ for any $s_{j, k}$ and $t_{j, k}$ satisfying $\left(\frac{1}{s_{j, k}}, \frac{t_{j, k}}{s_{j, k}}\right) \in\left[a^{-j}, a^{-j+1}\right] \times\left[a^{-j} k b, a^{-j}(k+1) b\right]$. 
Proof of Theorem 2.3. Let $a, b, \Delta$ be defined as in Lemma 3.5. Take some integer $n_{1}>\frac{1}{a-1}$. Let

$$
n_{2 l}=\left\lfloor a n_{2 l-1}\right\rfloor, \quad n_{2 l+1}=\left\lceil a n_{2 l}\right\rceil, \quad l \geq 1 .
$$

It is easy to check that $n_{l+1}>n_{l}$ for any $l \geq 1$ and $n_{2 l}-n_{2 l-1}>(a-1) n_{2 l-1}-1 \rightarrow$ $+\infty$ as $l \rightarrow+\infty$. Let

$$
\Lambda=\left\{k \in \mathbb{Z}: k \leq n_{1} \text { or } n_{2 l} \leq k \leq n_{2 l+1} \text { for some } l \geq 1\right\}
$$

Then we have $D^{-}\left(a^{-1} b \Lambda\right)=0$. To prove this theorem, it suffices to prove that $\left\{\tau\left(a^{j-1}, a^{-1} b k\right) \psi: j \in \mathbb{Z}, k \in \Lambda\right\}$ is a frame for $L^{2}(\mathbb{R})$.

For any $k \in \mathbb{Z}$, if $k \notin \Lambda$, then there is some $l \geq 1$ such that $n_{2 l-1}<k<n_{2 l}$. Since $\frac{n_{2 l}}{a} \leq n_{2 l-1}<n_{2 l} \leq \frac{n_{2 l+1}}{a}$ thanks to (3.10), there is a unique $m_{k} \in\left[n_{2 l}, n_{2 l+1}\right] \cap \Lambda$ such that

$$
\frac{m_{k}}{a} \in\left(k, k+\frac{1}{a}\right] \subset(k, k+1) .
$$

Obviously, $m_{k} \neq m_{k^{\prime}}$ if $k \neq k^{\prime}$. Let

$$
\left(s_{j, k}, t_{j, k}\right)= \begin{cases}\left(a^{j-1}, a^{-1} b k\right), & j \in \mathbb{Z}, k \in \Lambda, \\ \left(a^{j}, a^{-1} m_{k} b\right), & j \in \mathbb{Z}, k \notin \Lambda .\end{cases}
$$

Then $\left(\frac{1}{s_{j, k}}, \frac{t_{j, k}}{s_{j, k}}\right) \in\left[a^{-j}, a^{-j+1}\right] \times\left[a^{-j} b k, a^{-j} b(k+1)\right]$ for any $j, k \in \mathbb{Z}$. It follows from Lemma 3.5 that

$$
\begin{aligned}
& 2 \sum_{j \in \mathbb{Z}, k \in \Lambda}\left|\left\langle f, \tau\left(a^{j-1}, a^{-1} b k\right) \psi\right\rangle\right|^{2} \\
= & \sum_{j \in \mathbb{Z}, k \in \Lambda}\left|\left\langle f, \tau\left(a^{j-1}, a^{-1} b k\right) \psi\right\rangle\right|^{2}+\sum_{j \in \mathbb{Z}, k \in \Lambda}\left|\left\langle f, \tau\left(a^{j}, a^{-1} b k\right) \psi\right\rangle\right|^{2} \\
\geq & \sum_{j, k \in \mathbb{Z}}\left|\left\langle f, \tau\left(s_{j, k}, t_{j, k}\right) \psi\right\rangle\right|^{2} \geq \frac{1}{2(a-1) b}\left(C_{\psi}^{\frac{1}{2}}-\Delta\right)^{2}\|f\|^{2}, \quad \forall f \in L^{2}(\mathbb{R}) .
\end{aligned}
$$

Since $\Lambda \subset \mathbb{Z}$, using Lemma 3.5 again, we get $\sum_{j \in \mathbb{Z}, k \in \Lambda}\left|\left\langle f, \tau\left(a^{j-1}, a^{-1} k b\right) \psi\right\rangle\right|^{2} \leq$ $\frac{a^{2}}{2(a-1) b}\left(C_{\psi}^{\frac{1}{2}}+\Delta\right)^{2}\|f\|^{2}$. Hence $\left\{\tau\left(a^{j-1}, a^{-1} k b\right) \psi: j \in \mathbb{Z}, k \in \Lambda\right\}$ is a frame for $L^{2}(\mathbb{R})$.

To conclude this paper, let us check Example 2.1 Since $\hat{\psi}$ is absolutely continuous and compactly supported, it is easy to see that $\psi$ meets the hypotheses of Theorem 2.3. Moreover, it can be checked that

$$
C_{\psi}=\frac{32}{15}, \quad C_{\psi^{\prime}}=\frac{32}{315}, \quad C_{\tilde{\psi}}=\frac{6}{5}, \quad C_{(\tilde{\psi})^{\prime}}=\frac{18}{35},
$$

and $\Delta<C_{\psi}^{\frac{1}{2}}$ for $a=2$ and $b=1$. Let $n_{l}=2^{l}, l \geq 1$. Then (3.11) turns out to be $\Lambda=\left\{k \in \mathbb{Z}: k \leq 2\right.$ or $2^{2 l} \leq k \leq 2^{2 l+1}$ for some $\left.l \geq 1\right\}$. Hence $\left\{\tau\left(2^{j-1}, \frac{k}{2}\right) \psi: j \in \mathbb{Z}, k \in \Lambda\right\}$ and so $\left\{\tau\left(2^{j}, \frac{k}{2}\right) \psi: j \in \mathbb{Z}, k \in \Lambda\right\}$ are frames for $L^{2}(\mathbb{R})$.

\section{ACKNOWLEDGMENTS}

We thank the referee very much for valuable and elaborate suggestions which helped us to improve this paper. 


\section{REFERENCES}

[1] J. J. Benedetto, Irregular sampling and frames, in "Wavelets: A Tutorial in Theory and Applications (C. K. Chui, editor), Academic Press, Boston, 1992, pp. 445-507. MR 93c:42030

[2] A. Beurling, Balayage of Fourier-Stieltjes transforms, The Collected Works of Arne Beurling, Vol. 2, Harmonic Analysis, L. Carleson, P. Malliavin, J. Neuberger, and J. Wermer, editors, Birkhäuser, Boston, 1989, pp. 341-365. MR 92k:01046b

[3] O. Christensen, Frames, Riesz bases, and discrete Gabor/wavelet expansions, Bulletin (new series) of Amer. Math. Soc., 38 (2001), 273-291. MR 2002c:42040

[4] O. Christensen, B. Deng, and C. Heil, Density of Gabor frames, Appl. Comput. Harmon. Anal., 7 (1999), 292-304. MR 2000j:42043

[5] C. K. Chui and X. L. Shi, Orthonormal wavelets and tight frames with arbitrary real dilations, Appl. Comput. Harmon. Anal., 9 (2000), 243-264. MR 2002a:42025

[6] I. Daubechies, The wavelet transform, time-frequency localization and signal analysis, IEEE Trans. Inform. Theory, 36 (1990), 961-1005. MR 91e:42038

[7] I. Daubechies, Ten Lectures on Wavelets, SIAM, Philadelphia, 1992. MR 93e:42045

[8] H. Feichtinger and K. Gröchenig, Banach spaces related to integrable group representations and their atomic decompositions, I, J. Funct. Anal., 86 (1989), 307-340. MR 91g:43011

[9] K. Gröchenig, Describing functions: atomic decompositions versus frames, Monatsh. Math., 112 (1991), 1-42. MR 92m:42035

[10] K. Gröchenig, Irregular sampling of wavelet and short-time Fourier transforms, Constr. Approx., 9 (1993), 283-297. MR 94m:42077

[11] G. H. Hardy, J. E. Littlewood, and G. Pólya, Inequalities, 2nd Ed., Cambridge Univ. Press, Cambridge, 1952. MR 13:727e

[12] C. Heil and G. Kutyniok, Density of weighted wavelet frames, J. Geom. Anal., 13 (2003), 479-493.

[13] C. Heil and D. Walnut, Continuous and discrete wavelet transforms, SIAM Review, 31 (1989), 628-666. MR 91c:42032

[14] S. Jaffard, A density criterion for frames of complex exponentials, Michigan Math. J., 38 (1991), 339-348. MR 92i:42001

[15] P. Olsen and K. Seip, A note on irregular discrete wavelet transforms, IEEE Trans. Inform. Theory, 38 (1992), 861-863.

[16] T. E. Olson and R. Z. Zalik, Nonexistence of a Riesz basis of translates, in "Approximation Theory", pp. 401-408, Lecture Notes in Pure and Applied Math., Vol. 138, Marcel Dekker, New York, 1992. MR 93b:41001

[17] W. Sun and X. Zhou, Irregular wavelet frames, Science in China, series A, 43 (2000), 122127. MR 2000m:42035

[18] W. Sun and X. Zhou, Irregular wavelet/Gabor frames, Appl. Comput. Harmon. Anal., 13 (2002), 63-76. MR 2003i:42057

[19] W. Sun and X. Zhou, Density and stability of wavelet frames, Appl. Comput. Harmon. Anal., 15 (2003), 117-133.

[20] R. Young, An Introduction to Nonharmonic Fourier Series, Pure and Applied Mathematics, no. 93, Academic Press, New York, 1980. MR 81m:42027

[21] X. Zhou and Y. Li, A class of irregular wavelet frames, Chinese Science Bulletin, 42 (1997), $1420-1424$.

Department of Mathematics, Nankai University, Tianjin 300071, China

E-mail address: sunwch@nankai.edu.cn

Department of Mathematics, Nankai University, Tianjin 300071, China

E-mail address: xwzhou@nankai.edu.cn 